EPJ manuscript No.

(will be inserted by the editor)

\title{
Fluid and plastic flow dynamics of the critical state for a strongly pinned 2D superconductor
}

D. Monier and L. Fruchter

Laboratoire de Physique des Solides, C.N.R.S. Universit Paris-Sud, 91405 Orsay cedex, France

Received: date / Revised version: date

\begin{abstract}
We present simulations of the dynamic critical state for a $2 \mathrm{D}$ superconductor with strong pinning centers, corresponding to a matching field twice the applied magnetic field. The sharp crossover between the plastic regime, at low current density and temperature, and the fluid flow regime for flux motion is characterized by the activation energy for flux motion and the transverse diffusion of the vortices trajectory.
\end{abstract}

PACS. 64.60.Ht Dynamic critical phenomena - 74.60.Ge Flux pinning, flux creep and flux-line lattice dynamics

\section{Introduction}

Flux pinning in type II superconductors is a well documented example for the general problem of an elastic periodic medium interacting with disorder. The study of the dynamics of the pinned flux line lattice, under the action of an external current or by the virtue of a time varying external magnetic field, is one way to apprehend the problem of flux line pinning. Well known examples are transport I-V characteristics and magnetization relaxation experiments. Such experiments are usually interpreted using simple models, assuming a uniform critical current density and a single, current dependent, activation energy at non zero temperature. However, numerical simulations]四,

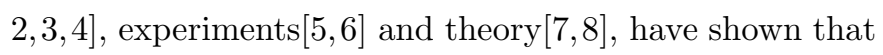
a driven flux lattice can be a rather complex object, remarkably different from the simple pictures used in the interpretation of the experiments. In particular, the pinning of a two-dimensional flux lattice by strong pinning centers lends itself easily to numerical simulations and has been the subject of several studies. Such a system is also a good approximation for highly anisotropic superconductors irradiated by heavy ions, such as the high Tc oxides, which are now well documented. In this paper, we present numerical simulations of this strong pinning situation, at 
non zero temperature and for conditions similar to those encountered in experimental magnetization experiments.

\section{Simulation}

We simulate a slab submitted on one side to an external magnetic field, applied parallel to its boundary, while, on the opposite side, the external magnetic field is assumed to be zero (vortices are removed as they cross this boundary). Periodic conditions are used, so that the effect of the finite dimensions is limited. Flux lines are assumed rigid rods. This correctly modelizes a layered superconductor with decoupled layers in the critical state, for which the local magnetic induction decreases from the applied field value on the external sides to a lower value at the center of the sample (Fig. 1).

Strong pinning centers are randomly distributed in the sample. The density of the pinning sites is $n=B_{\Phi} / \Phi_{0}$, with $\Phi_{0}$ the flux quantum and $B_{\Phi}$ the 'matching field' for which an equilibrium flux line lattice shows the same density of flux lines. A region of width $\lambda$ from the side where vortices are introduced is kept free of pinning sites, in order to allow for the initial formation of a regular hexagonal lattice in this region. The pinning sites are assumed normal cylinders parallel to the applied field, with radius $c_{0}$. The situation where, at low temperature, the vortex core radius $\xi(T)$ is smaller than $c_{0}$ and pinning is due essentially to the reduction of the core energy when the line sits on the pin is considered. The force exerted by a pin at a distance $r$ to the line is given by:

$$
f_{p}(r)=\left\{\begin{array}{l}
\sigma \varepsilon_{o} r / r_{0} \xi \text { for } r \leq r_{0} \\
0 \text { for } r>r_{0}
\end{array}\right.
$$

where $\varepsilon_{0}=\left(\Phi_{0} / 4 \pi \lambda\right)^{2}$ is the line energy, $r_{0}=c_{0}+\xi / 2$ and $\sigma \leq 1$.

The force per unit length exerted by a vortex at a distance $r$ to the line is:

$$
f_{v v}(r)=\left(\Phi_{0}^{2} / 8 \pi^{2} \lambda^{3}\right) K_{1}(r / \lambda)
$$

where $K_{1}$ is a Bessel function. This is a good approximation strictly only in the case of vortex lines (rods) and for $2 \mathrm{D}$ vortices a logarithmic interaction should be used. In the present case, the more rapid decrease of the Bessel function allows us to cut the interaction between vortices at a distance $5 \lambda$ and save computation time.

The external magnetic field $B_{0}$ is simulated by an extra force $f_{B_{0}}$ acting on each vortex, perpendicular to the external side of the sample. The force acting on a vortex at a distance $x$ from the boundary is the one imposed by a semi-infinite vortex lattice at a distance $a_{0}+x$, where $a_{0}=\left(\Phi_{0} / B\right)^{1 / 2}$ is the flux lattice spacing at the equilibrium.

The finite temperature is simulated in a way similar to the one described in ref. [9] by adding a stochastic velocity to the particles:

$$
\mathbf{v}_{\mathbf{T}}=\left(2 k_{B} T \tau / 3 \Delta^{2}\right)^{1 / 2}\left(\begin{array}{c}
\gamma_{x} \\
\gamma_{y}
\end{array}\right) \Theta(\Delta t / \tau-q)
$$

where $1 / \tau$ is the average frequency for the thermal perturbation, $\gamma_{x, y}$ are random numbers from a Gaussian distribution of width $1, q$ is a random number from a uniform 
distribution between 0 and 1 , and $\Theta$ is the Heaviside function. We have used a single set of parameters $\tau$ and $\Delta t$ for all temperatures with $\tau \gg \Delta t$. The accuracy for the temperature simulated in this way was checked by computing the life time of a single vortex in a potential well similar to the ones defined above. The life time as a function of the well depth was found to match accurately the one given by the Arrhenius-Kramers relation for an overdamped particle, in the range of temperature studied here. In this overdamped regime, results are independent of the viscosity when expressed in the time unit $t / \eta$, the escape frequency being inversely proportional to $\eta$.

Finally, the displacement $\Delta \mathbf{r}_{\mathbf{i}}$ of vortex $i$ during time step $\Delta t$ is computed according to the diffusive equation:

$$
\begin{aligned}
& \eta \Delta \mathbf{r}_{\mathbf{i}} / \Delta t= \\
& \sum_{j \neq i} \mathbf{f}_{\mathbf{v v}}\left(r_{i j}\right)+\sum_{j} \mathbf{f}_{\mathbf{p}}\left(r_{i j}\right)+\mathbf{f}_{\mathbf{B}_{\mathbf{0}}}\left(x_{i} / a_{0}\right)+\eta \mathbf{v}_{\mathbf{T}}
\end{aligned}
$$

where the first sum is over all vortices at a distance less than $5 \lambda$ and the second one is over all pins ( $\eta$ is the viscosity per unit length). The time step is chosen so that the vortex displacement during one step is always much smaller than the pinning characteristic length, $r_{0}$. At non zero temperature, vortices tend to creep through the sample from the boundary submitted to the external field, where vortices are fed whenever the magnetic pressure is strong enough to allow for their penetration, to the one with zero applied field. As a consequence, a steady thermally activated flux flow is set after some time, characterized by a linear flux density profile and a steady vortex current density, $\mathcal{F}$, that flows through the sample.

\section{Results}

In the rest, we use the following material parameters, appropriate for a high temperature superconductor: $\lambda=$ $1400 \AA, \xi=18 \AA, c_{0}=35 \AA$ and $\sigma=0.1$. The samples held typically 5000 pinning sites and 1000 vortices in the steady state. The matching field, $B_{\Phi}=5000 G$ and the external field, $B_{0}=2500 G$, were the same for all the simulations.

In a way similar to what is done in the analysis of real relaxation experiments, we use a one-dimensional model with a single activation energy to analyze the vortex current density 10]. According to this widely used model, vortices thermally hop over barriers disposed along a 1$\mathrm{D}$ axis with height $U(j)$ (where $j=(4 \pi)^{-1} \partial B / \partial x$ is the current density) and the vortex current density is $\mathcal{F}=j B \phi_{0} \eta^{-1} \exp \left(-U(j) / k_{B} T\right)$. This model clearly misses some of the fundamental features of the real experiments. As pointed in ref. [10], it is valid only in the case of almost uniform vortex and current densities, when physical quantities may be replaced by their average over several vortex spacing. In particular, it assumes that the screening current flows in our case along straight lines perpendicular to the vortex flow. As can be seen in Fig. 3A, this is not so and the current tends to flow along some curved, branched paths that reveal the highly inhomogeneous stresses in the pinned flux lattice. There has been attempts to account for the spatial heterogeneity of the real systems, using a distribution of activation energies 11 . 12. However, the method assumes parallel, independent relaxation channels, which is still a very crude assump- 
tion. As for real magnetization experiments analysis, we obtain the average screening current density from the total magnetization of the sample. In order to investigate different flux gradient profiles, different sample geometries were used with the length of the cylinder between $20 \lambda$ and $100 \lambda$ and diameters between $10 \lambda$ and $300 \lambda$. The experiments probed different $T$ and $j$ values, which are located on a smooth $(T, j)$ trajectory shown in Fig.2. This is similar to relaxation rate measurements in superconductors, for which both the temperature and the screening current are varied - the latter parameter being implicitly determined by the temperature and the time window explored in these experiments.

The activation energy obtained from the simulations is shown in Fig. 2, where energy is normalized to the single vortex pinning site energy, $U_{0}=\sigma \varepsilon_{0} r_{0} / \xi$, and critical current density to the single vortex critical current, $j_{0}=\sigma \varepsilon_{0} / \xi \phi_{0}$. It is shown as a $3 \mathrm{D}$ plot, as the computation of the energy with one of these two parameters fixed would imply prohibitive computation time as temperature or current decreases. The same limitation is also encountered in real relaxation experiments, although in this case the temperature variations are usually disregarded and the data plotted as $U(j)$. The reason for this is that Maley's procedure 13] allows to show that, in most cases, the activation energy obtained from relaxation experiments is temperature-independent. We notice, however, that this is no longer true at low temperature, which has been attributed to the occurrence of the quantum tunneling regime 14 .
A clear upturn can be seen in Fig. 2, where the activation energy increases strongly with decreasing current below $j / j_{0} \simeq 0.05, k_{B} T / \Delta \simeq 0.25$. This upturn corresponds to the crossover between a plastic to a fluid flow regime for flux motion. This can be seen, first, from a direct observation of the vortices trajectories: in the plastic regime, there exists a time scale at which some vortices move over distances larger than $a_{0}$, while others remain immobile on that scale and constitute some kind of 'pinned islands' (Ref.[2]). It is important to stress that these islands depin at a larger time scale for non zero temperature, so that only a dynamical definition of these domains may be given in our case. No such characteristic time may be found in the fluid flow regime where, depending on the time scale considered, nearly all or none of the vortices move over this distance (Fig. 3A,B). A more quantitative argument in favor of two clearly distinct regimes is given by the examination of the diffusion of a vortex trajectory, transverse to the average flux flow (Fig. 4 and 5). In the fluid flow regime, the transverse displacement on the scale of the equilibrium flux lattice parameter, $\delta=a_{0}^{-1}\left\langle\left[y(0)-y\left(a_{0}\right)\right]^{2}\right\rangle^{1 / 2}$ where the average is performed along the vortex trajectory, is significantly smaller than 1 , whereas it increases with decreasing temperature and increasing current density up to a value $\simeq 0.6-0.65$ in the plastic regime (Fig. 5). This limiting value may be understood by considering that the most disordered trajectory allowed in the flux lattice is the one where a vortex hops randomly from one interstitial site to a neighbor site. A consequence of disorder in the plastic regime is that the 
examination of the flux pattern hardly reveals the orientation of the average flux flow (Fig. $3 \mathrm{C}$, making abstraction of the region close to the zero field boundary of the sample, where side effect is visible), as compared to the fluid flow regime (Fig. 3B). As can be seen in Fig. 司, the increase of the transverse displacement along the $(T, j)$ trajectory shown in Fig. 20 is rather abrupt, suggesting that the trajectory might cross a transition line rather than a crossover line. If this is so, an order parameter may be elaborated from $\delta$, after subtraction of the high temperature baseline and adequate normalization, which is unity in the ordered regime and tends towards zero in the opposite limit, thus delimiting two distinct, nonequilibrium, phases. The static topological orders of the two phases which would be defined in this way do not show significant differences: both lack long range order, either crystal-like or smectic-like. To that respect, they both look like a liquid, as demonstrated by the inspection of the density autocorrelation function (Fig. 6). It is difficult, however, to unambiguously put into evidence the existence of a second-order nonequilibrium phase transition in our case.

Up to now, we have reported only upon a single point of the crossover line in the $(T, j)$ plane. A close examination of Fig. 3 provides some indication about the general behavior of this line. Indeed, it can be seen that both samples $\mathrm{B}$ and $\mathrm{C}$ do not exhibit a strictly homogeneous regime and that the region close to their bottom boundary tends to be in the fluid flow regime. This is because, due to edge effects, the average screening current density tends to be larger in this region. As a consequence, we may now propose a schematic phase diagram for flux motion, where we have added the disorder strength as a third dimension. These observations are not easily compared to the theoretical expectations for driven lattices ([8] and refs therein). First, models investigate the case of a weak random potential, which is not adequate here, unless the matching field greatly exceeds the applied magnetic field and $\sigma \ll 1$. Then, the two dimensional, non zero temperature case for the random potential is far less documented than the three dimensional one[8]. We may simply notice that the measured activation energy at the crossover is close to the plastic energy barrier (i.e. the barrier experienced by a moving interstitial defect) $U_{p l} \simeq \varepsilon_{0} / 2 \sqrt{3} \pi \simeq 0.4 U_{0}$ (Fig. 2) 16 and that it is likely that this condition holds all along the crossover line. Indeed, energy barriers larger than $U_{p l}$ cannot be allowed by decoupled vortex flow channels and larger barriers can be sustained only by the coupling of these channels in the fluid flow regime. Finally, we would like to compare with real relaxation experiments. The main difficulty in realizing the situation described by our simulations comes from the presence, in real crystals, of naturally grown point defects. Indeed, as-grown crystals invariably exhibit at low temperature high critical current. In the highly anisotropic $\mathrm{Bi}_{2212}$ material, screening current as large as $10^{10} \mathrm{Am}^{-2}$ is observed at low temperature. The introduction of strong pinning centers, such as columnar defects, does however induce a sizeable increase of flux line pinning at low temperature (almost one order of magnitude for the critical current density at $4.2 \mathrm{~K}$ in 
Ref. 17], at a dose $B_{\phi}=20 k G$ ). From this, it may be concluded that natural defects are weak or dilute enough, so as to be overcome by the introduction of extra columnar defects, and that irradiated crystals might provide a situation close to the one described here. An expected signature for the crossover is a weaker temperature dependence of the (relaxed) screening current in the fluid flow regime as compared to the plastic one - due to the increase of the energy barrier in this regime. A sharp change is indeed observed in the temperature dependence of the relaxed screening current, for both as-grown and irradiated samples, although this has been interpreted as the signature of the crossover between small and large bundles regime in the first case, and depinning from the defects in the second one 17. We believe that a decisive test should be the measurement of the energy barriers at this point and the comparison with the one for the plastic mechanism.

\section{Conclusion}

We have studied the crossover between the fluid flow and the plastic regimes of the critical state dynamics for a two dimensional flux lattice, with strong pinning sites corresponding to a matching field twice the applied field. Following a smooth trajectory in the $(T, j)$ diagram, we find that the transverse displacement of the vortex trajectory rises abruptly as this trajectory crosses the crossover line. There, the apparent activation energy for flux motion is close to the plastic energy barrier and increases strongly in the fluid flow regime. We point out strong similarities between these results and the sharp crossover observed in the temperature dependence of the screening current density for as-grown and irradiated $\mathrm{Bi}_{2212}$.

\section{References}

1. H.J. Jensen, A. Brass, An-Chang Shi and J. Berlinsky, Phys. Rev. B. 41, 6394 (1990).

2. F. Nori, Science 271,(1996) 1373.

3. C. Reichhardt, C.J. Olson, J. Groth, S. Field and F. Nori, Phys. Rev. B 53,(1996) R8898.

4. A.B. Kolton, D. Dominguez and N. Gronbech-Jensen, Phys. Rev. Lett. 83, (1999) 3061.

5. T. Matsuda, K. Hirada, H. Kasai, O. Kaminura and A. Tonomura, Science 271,(1996) 1393.

6. M. Marchevsky, J. Aarts, P.H. Kes and M.V. Indenbom, Phys. rev. Lett. 78,(1997) 531.

7. T. Giamarchi and P. Le Doussal, Phys. Rev. Lett. 76, (1996) 3408.

8. P. Le Dousssal and T. Giamarchi, Phys. Rev. B 57, (1998) 11356.

9. A. Brass and H.J. Jensen, Phys. Rev. B 39, (1989) 9587.

10. C.J. van der Beek, G.J. Nieuwenhuys, P.H. Kes, H.G. Schnack and R. Griessen, Physica C 197, (1992) 320.

11. R. Griessen, Physica C 175, (1991) 315.

12. A. F. Hoekstra, A. M. Testa, G. Doornbos, J. C. Martinez, B. Dam, R. Griessen, B. I. Ivlev, M. Brinkmann, K. Westerholt, W. K. Kwok and G. W. Crabtree, Phys. Rev. B 59, (1999) 7222.

13. M. P. Maley, J. O. Willis, H. Lessure and M. McHenry, Phys. Rev. B 42, (1990) 2639.

14. D. Monier and L. Fruchter 58,(1998) R8917.

15. D.J. Bishop, P.L. Gammel, C.A. Murray, D.B. Mitzi and A. Kapitulnik, Physica B 169, (1991) 72. 
16. G. Blatter, M.V. Feigel'man, V.B. Geshkenbein, A.I. Larkin and V.M. Vinokur, Rev. Mod. Phys. 66,(1994) 1125, chap. VIII.

17. V.V. Moshchalkov, V.V. Metlushko, G. Gntherodt, I.N. Goncharov, A. Yu. Didyk and Y. Bruynseraede, Phys. Rev. B 50,(1994) 639.
Fig. 1. Geometry used for the simulations. The average vortex flow is along the $x$ direction. Dotted line depicts the periodic boundary condition.

Fig. 2. Top: $(T, j)$ trajectory followed during the simulations. Bottom: activation energy along the $(T, j)$ trajectory. The dotted line indicates the plastic barrier, $U_{p l}$. Labels 'B' and 'C' refer to the samples in Fig.3.

Fig. 3. Sample A: static 'critical state' at $T=0$. Lines are colinear to the local current direction and their length indicate the current intensity (non linear scale). Samples B and C: same notation as in Fig. 2; lines are vortices trajectories in the steady state.

Fig. 4. Displacement transverse to the average velocity, along vortices trajectories, $y(x)$ (the average vortex flow is along the $x$-axis).

Fig. 5. The transverse displacement on the scale of the equilibrium lattice parameter. Lines are guides to the eye.

Fig. 6. Left: in gray scale, density autocorrelation function of the lattice snapshots. Right: after radial integration. Top and bottom are respectively sample B and C in Fig. A, both showing liquid order (The exponential decay length, as defined in Ref. 15, is respectively $0.8 a_{0}$ and $0.5 a_{0}$ ).

Fig. 7. Schematic phase diagram. The arrow indicates the trajectory followed in Fig. 2, with indication of samples B and $\mathrm{C}$ in Fig. 2 and 3. 


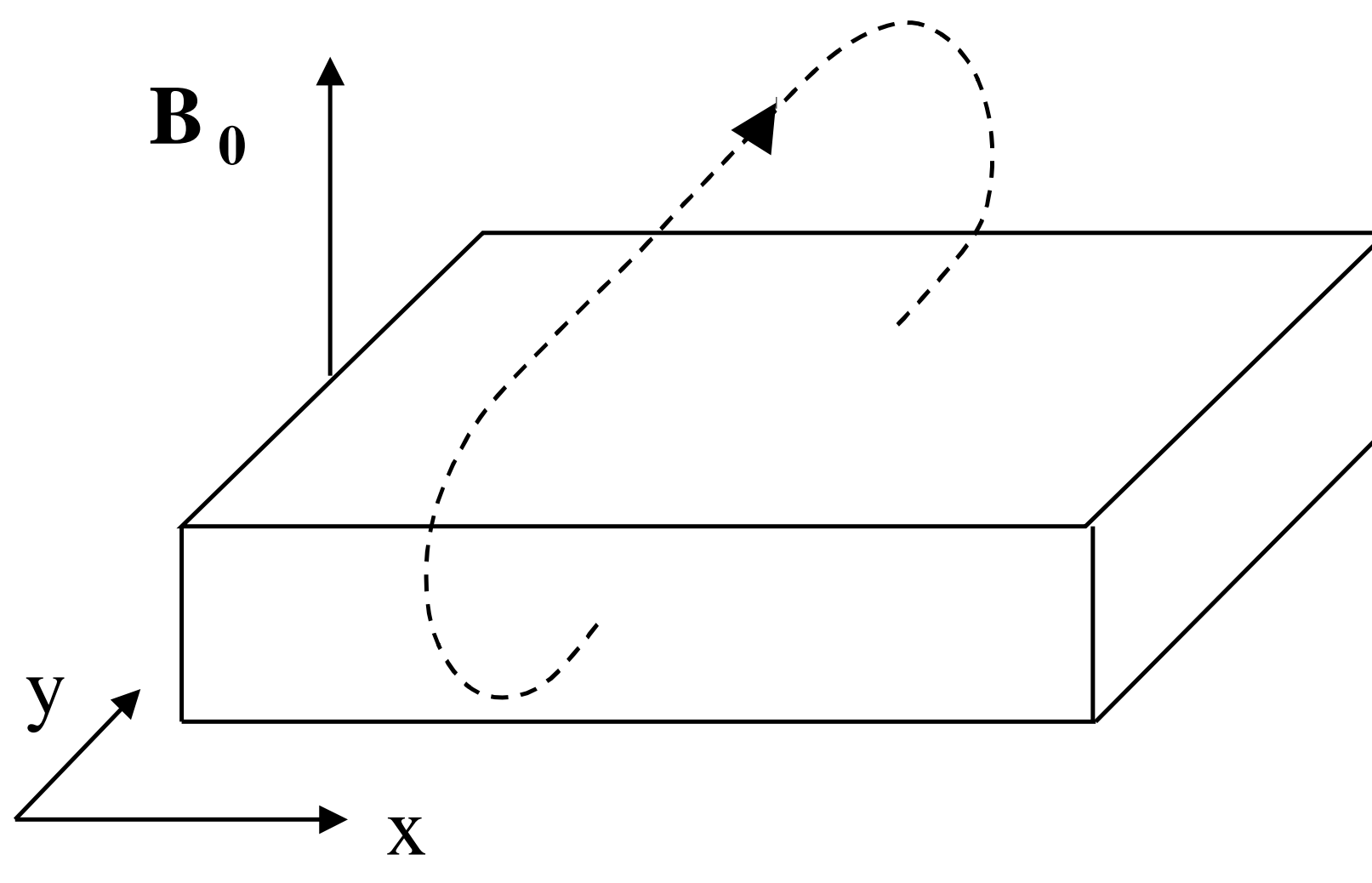



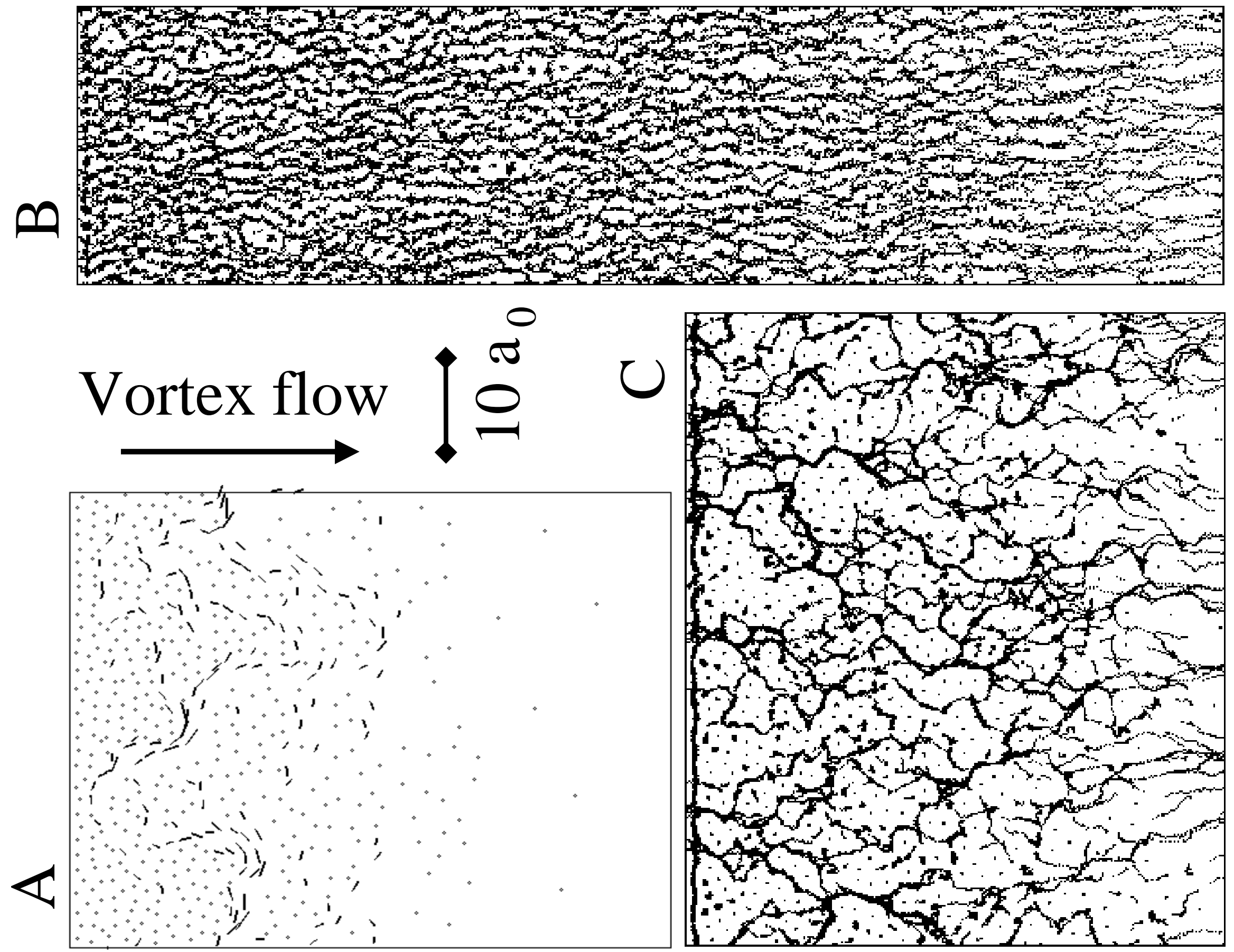


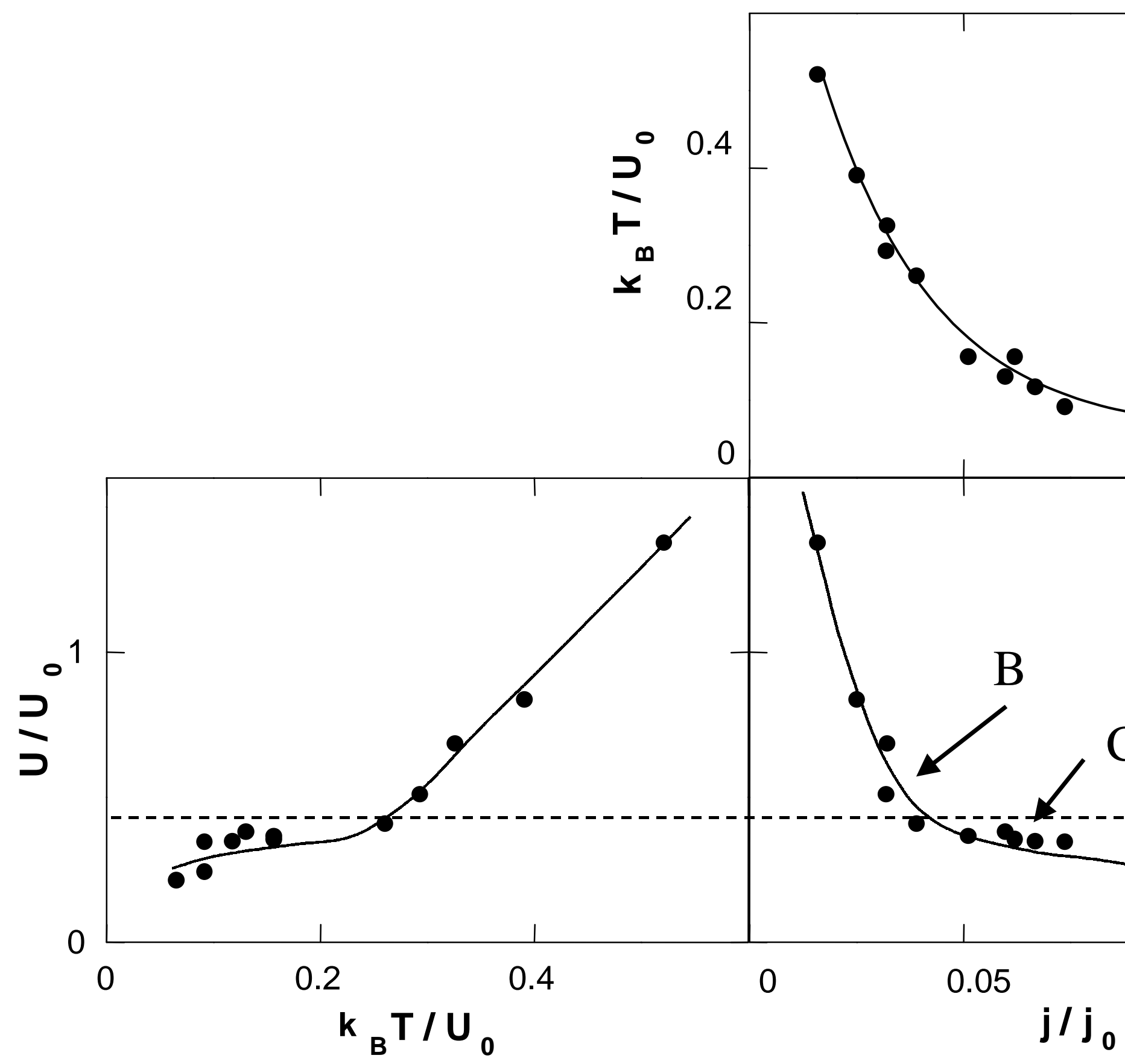




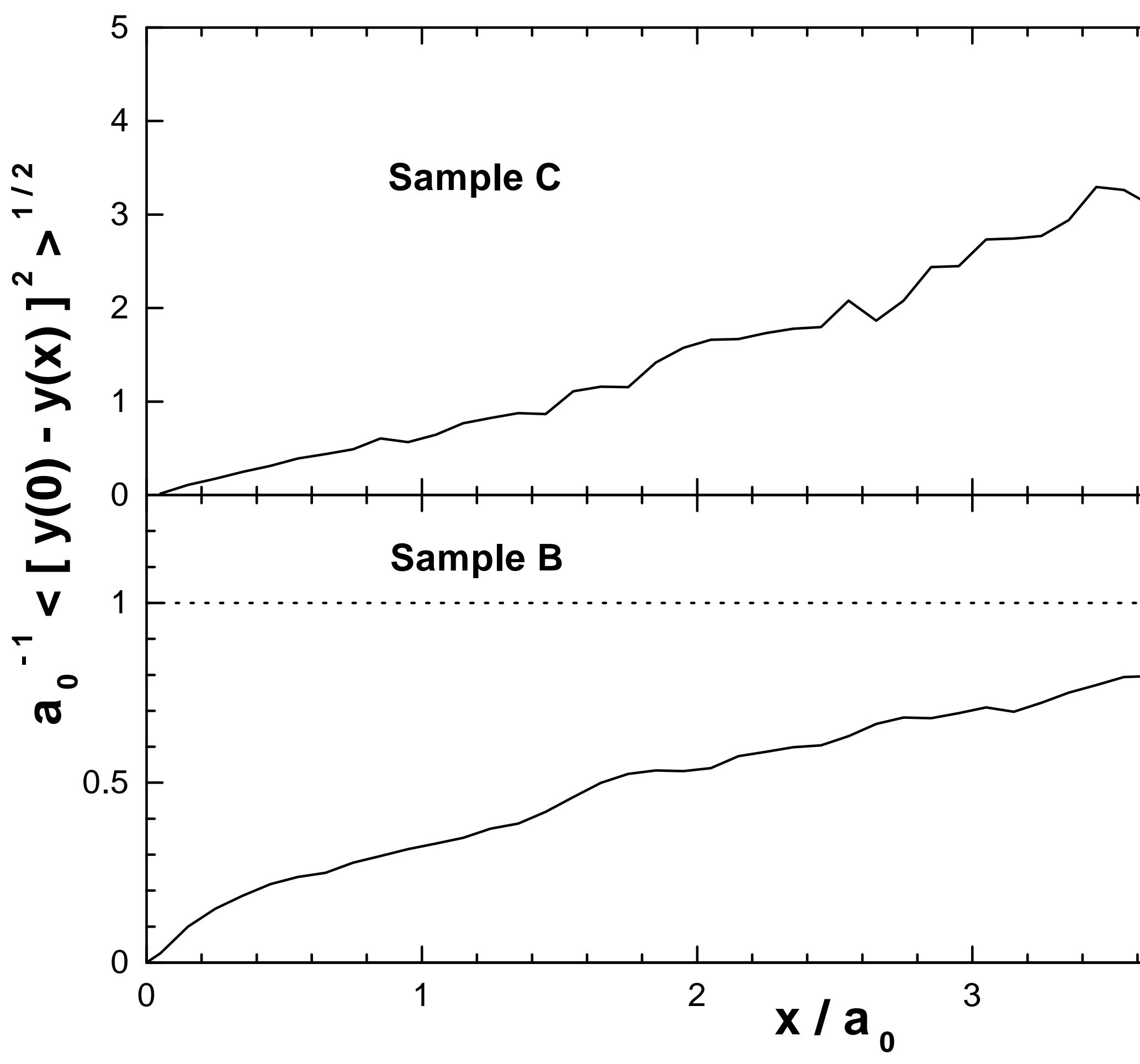




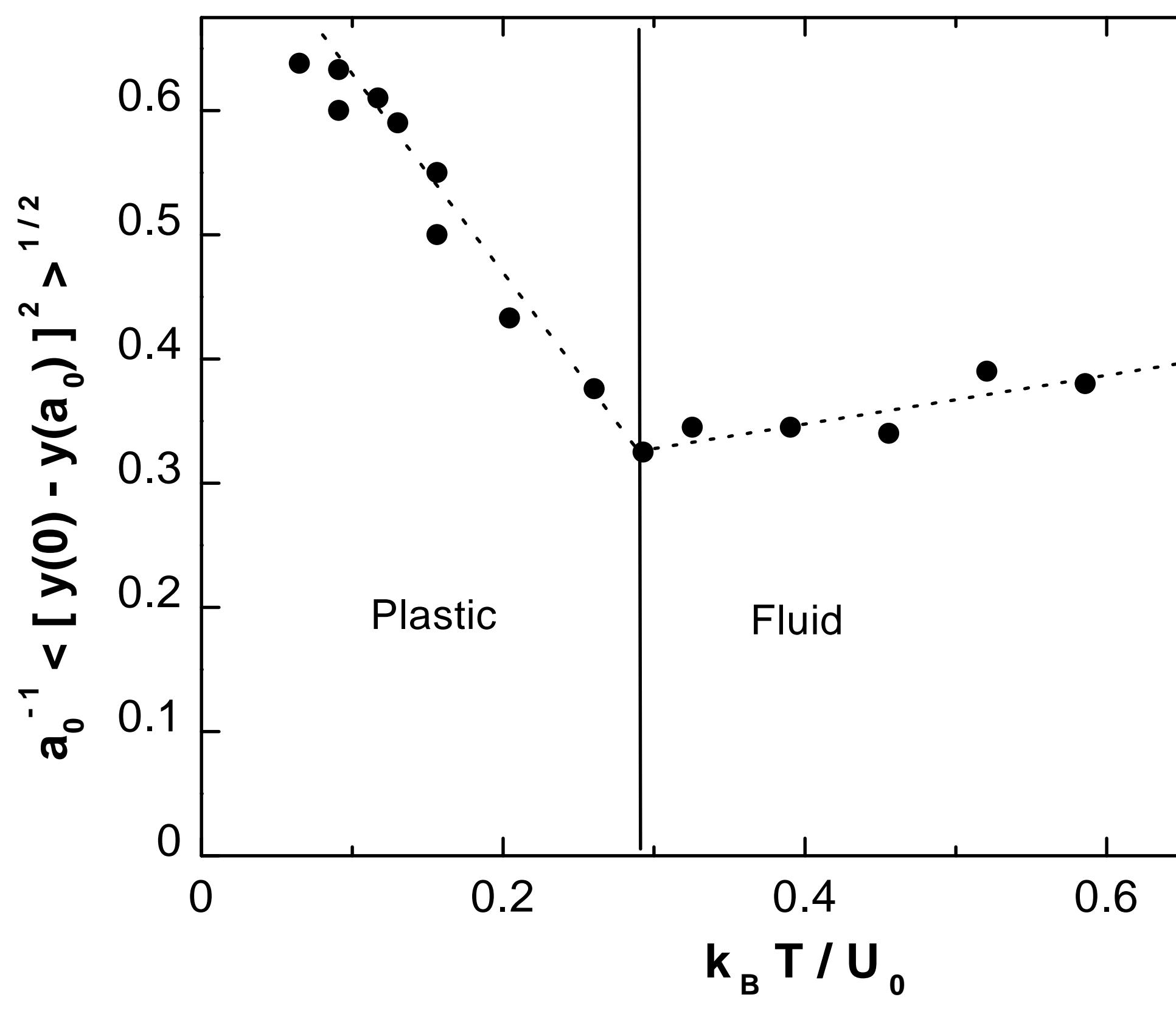


This figure "fig6.GIF" is available in "GIF" format from: http://arxiv.org/ps/cond-mat/0006340v2 


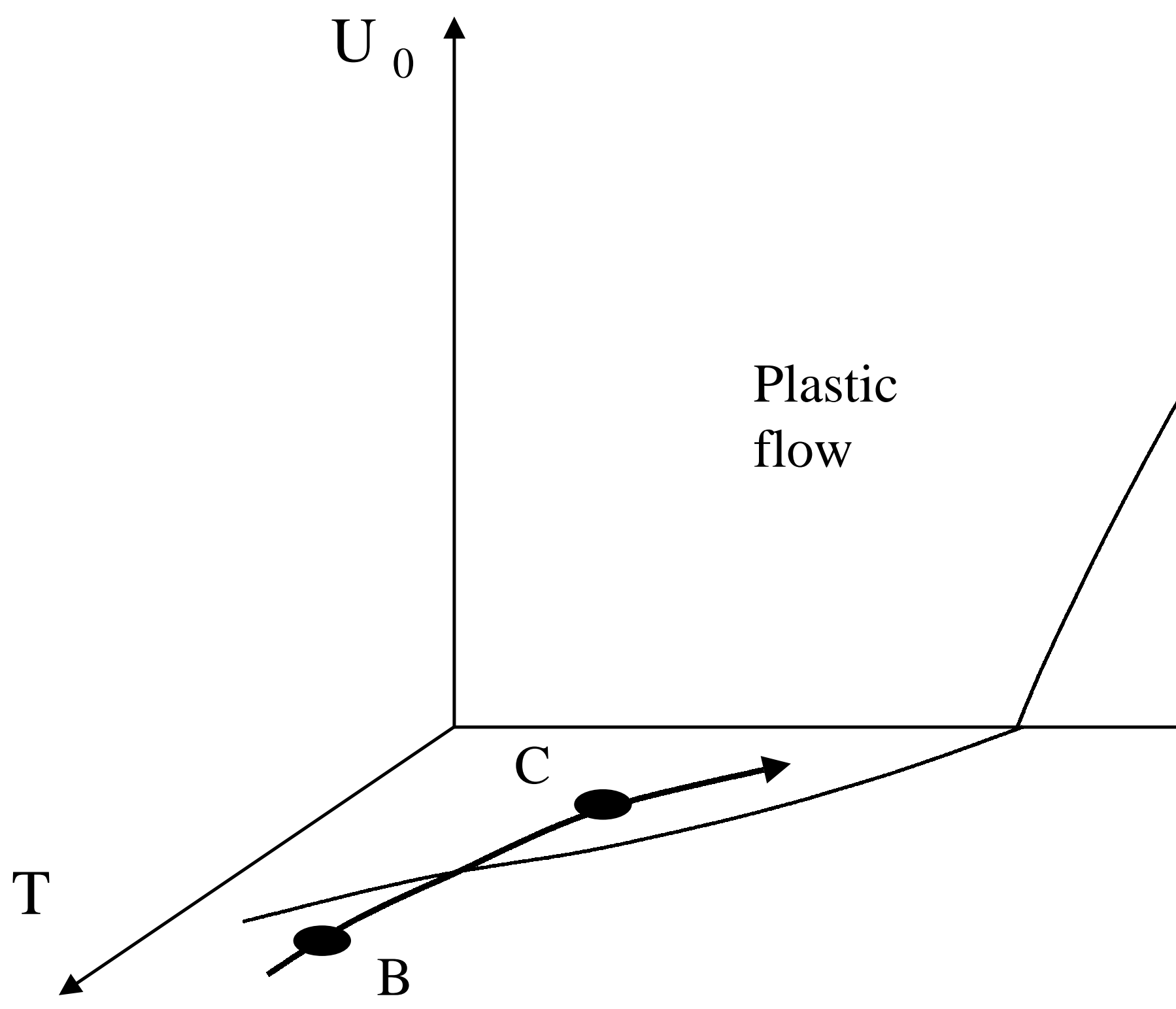

